\title{
A Survey Assessing Kansas Physician Assistants' Attitudes/Beliefs and Current Practices Regarding Implementation of Fall Prevention Strategies in Older Adults
}

\author{
LaDonna S. Hale, Pharm.D. ${ }^{1}$, Shelton J. Fraser, MPA, PA-C ${ }^{1}$, Kayla R. Keuter, MPH, PA-C ${ }^{1}$,
} Felecia A. Lee, Ph.D. ${ }^{2}$, Gina M. Berg, Ph.D., MBA ${ }^{2,3}$

${ }^{1}$ Wichita State University

Department of Physician Assistant

${ }^{2}$ University of Kansas School of Medicine-Wichita

Department of Family and Community Medicine

${ }^{3}$ Wesley Medical Center, Trauma Services, Wichita, KS

\begin{abstract}
Background. Falls are the leading cause of injury death, nursing home placement, and hospital trauma admissions in older adults. Although guidelines to reduce falls have been available for over a decade, routine implementation by healthcare providers is less than optimal. The purpose of this study was to evaluate the attitudes/beliefs and current practices of Kansas physician assistants (PAs) regarding fall assessment/prevention strategies in older adults and barriers to implementing strategies into daily practice.

Methods. A 67-item survey was mailed to all 760 Kansas PAs in 2009; 152 responded. Logistic regressions were performed on current fall prevention practices (exercise, home safety, medications, and vision) to determine attitudes, beliefs, and barriers associated with implementation.

Results. Most PAs believe falls are preventable (87\%) and implementation of various prevention strategies are their professional responsibility (88\% - 96\%); yet, less than 50\% routinely implement them. Barriers included lack of time (27\%), lack of staff (26\%), and feeling illprepared (18\%). Multiple logistic regressions revealed correlations among implementing the medication review strategy and lack of time as well as practicing the exercise strategy and lack of time and awareness of local exercise programs.

Conclusions. PAs are aware of the importance of fall prevention, believe falls are preventable, and believe it is their professional responsibility to implement fall prevention strategies with their older adult patients. However, most do not implement strategies in their practice due to a variety of internal and logistical barriers. Fall prevention materials/tools that are practical, simple, inexpensive, and require little implementation time may overcome barriers.

KS J Med 2015;8(4):135-142.
\end{abstract}

\section{Introduction}

Falls are the leading cause of injury death, traumatic brain injury, nursing home placement, and hospital trauma admissions in older adults (aged 65 years and older)., Of those that fall, $20 \%$ to $30 \%$ suffer moderate to severe injuries with impaired mobility and independence. ${ }^{2}$ In 2011, approximately 2.4 million older adults were treated in emergency departments (EDs) for nonfatal fall-related injuries. ${ }^{3}$ These injuries can include fractures, head traumas, joint dislocations, and soft tissue injuries. In 2013, the direct cost of unintentional falls in older adults (adjusted for inflation) was \$34 billion. The average hospitalization cost for a fall injury is over $\$ 35,000 .^{3}$ 
The Centers for Disease Control and Prevention (CDC), the National Council on Aging (NCOA), and the American Geriatrics Society (AGS) have identified key areas to reduce falls in older adults including: 1) increasing physical activity and balance/strength training, 2) evaluating and resolving home safety issues, 3) reviewing and resolving medication issues, and 4) screening and referral of vision problems. $^{1,4,5}$ Exercise programs and home safety interventions as well as multifactorial interventions targeting multiple fall risk strategies have demonstrated a reduction in the number of falls. ${ }^{6}$

Although guidelines have been available for over a decade, routine implementation by healthcare providers is less than optimal. ${ }^{7,8}$ Barriers previously identified by physicians were divided into three distinct categories: 1) physician factors (e.g., awareness, competing risks, training), 2) logistic factors (e.g., transportation, time, reimbursement) and 3) physicians' perception of patient factors (e.g., reporting, attitudes towards medications, positive feedback). ${ }^{8}$ It is essential to identify barriers to implementing evidence-based practices and determine the most effective method for incorporating those strategies in patient encounters to reduce fall risk.

Barriers and reasons for underutilization have not been well studied among Physician Assistants (PAs). PAs provide a significant amount of primary and emergency care across the nation. This is particularly true among rural and underserved communities similar to those found across much of Kansas where the percentage of older adults is on the rise. Therefore, understanding Kansas PAs' perceptions and practices regarding fall prevention is an important step in developing approaches to increase implementation nationwide. The purpose of this study was to evaluate the attitudes/beliefs and practices of PAs in
Kansas regarding fall assessment and prevention in older adults and barriers to integrating currently recommended, evidence-based fall prevention strategies into daily practice to increase implementation.

\section{Methods}

Mailing addresses for all 760 certified Kansas PAs were obtained from the Kansas Board of Healing Arts. A 67-item survey was created to ascertain respondent characteristics, attitudes/beliefs, current practices, and barriers to implementing fall prevention strategies. Survey content was drafted based upon fall prevention consensus guidelines ${ }^{1,4,5}$ and previously published data regarding barriers to implementation. ${ }^{8} \quad$ Eleven questions ascertained respondent characteristics. Thirty-five questions assessed attitudes/beliefs using a 4-point Likert scale ranging from "strongly agree" to "strongly disagree"; and eight questions assessed current practices as: "never", "sometimes", "frequently", and "always". Six commonly cited barriers were listed and respondents were asked to "check all that apply". Additionally, several open-ended questions were included and learner preferences were asked. Approval was obtained from all appropriate institutional review boards.

Statistical Analysis. Descriptive characteristics are reported as percentages and means, as appropriate. Binary logistic regressions were performed on current fall prevention practices (exercise, home safety, medications, and vision) as the criterion (dependent) variables. Variables found to be uncorrelated via multicollinearity diagnostics (VIF < 10) were entered into the logistic regression model. ${ }^{9}$ Statistical significance was set at $p<0.05$. Statistical analyses were performed using SPSS for Windows, Version 20.0. ${ }^{10}$ 


\section{Results}

The response rate was 20\% (152/760). Demographics including education, primary practice setting, and population of primary practice setting are shown in Table 1. Fifty- one (51\%) percent of responding PAs reported that the majority (50\% or more) of their patients were 65 years or older.

Table 1. Demographics of responding Kansas PAs ( $\mathrm{n}=152)$.

\begin{tabular}{|c|c|c|}
\hline & Mean & SD \\
\hline Age & 41.7 & 12.7 \\
\hline \multirow[t]{2}{*}{ Years in Practice } & 11.3 & 9.5 \\
\hline & \# & Percent \\
\hline Female & 100 & $66 \%$ \\
\hline \multicolumn{3}{|l|}{ Education } \\
\hline Bachelor & 69 & $45 \%$ \\
\hline Masters & 73 & $48 \%$ \\
\hline Doctorate & 2 & $1 \%$ \\
\hline \multicolumn{3}{|l|}{ Primary Practice } \\
\hline Family Medicine & 60 & $40 \%$ \\
\hline Emergency Medicine & 17 & $11 \%$ \\
\hline Orthopedics & 19 & $13 \%$ \\
\hline Cardiology & 13 & $9 \%$ \\
\hline Internal Medicine & 12 & $8 \%$ \\
\hline Other $^{\mathrm{a}}$ & 27 & $18 \%$ \\
\hline \multicolumn{3}{|l|}{ Population of Primary Practice Setting } \\
\hline$<10,000$ & 37 & $24 \%$ \\
\hline $10,000-49,999$, not near a metro area & 13 & $9 \%$ \\
\hline 10,000-49,999, near a metro area & 15 & $10 \%$ \\
\hline $50,000-99,999$ & 12 & $8 \%$ \\
\hline $100,000-299,999$ & 21 & $14 \%$ \\
\hline$>300,000$ & 47 & $31 \%$ \\
\hline
\end{tabular}

Note: Percentages may not match total sample due to missing data; percentages may not total $100 \%$ due to rounding.

${ }^{a}$ Dermatology (2); geriatrics (1); obstetrics/gynecology (2); occupational (2); pediatrics (1); surgery (4); infectious disease (1); VA general medicine (1); ENT (1); oncology (1); pulmonary/critical care (2); trauma (2); urology (1); urgent care (1); residential rehabilitation (1); pain management (1); hematology (1).

Attitudes/Beliefs. Respondents agreed (agree or strongly agree) falls are a significant public health problem (99\%) and most falls are preventable (87\%). They believe falls are under-reported by older adults out of fear of losing independence (80\%), fall prevention programs should be funded by the state (64\%), and screening for fall risk should be as routine as screening for other medical problems such as cancer, high 
cholesterol, and diabetes (93\%). Table 2 shows attitudes/beliefs regarding the four fall prevention strategies: exercise, home safety, medications, and vision. The majority agreed these strategies are effective (95\% - 99\%). Respondents agreed they have a professional responsibility to implement them (88\% - 96\%) and are willing to implement them (85\% - 93\%). However, respondents' beliefs that patients would be compliant with their suggestions regarding these strategies were somewhat lower for exercise (47\%), home safety (69\%), and vision (84\%) as compared to medications (90\%). Twenty percent (20\%) agreed (agree or strongly agree) with the statement, "I assume the pharmacy will notify me if I order a drug that potentially increases fall risk", and $82 \%$ agreed or strongly agreed with the statement, "I would appreciate being notified by the pharmacy if I order a drug that is known to potentially increase fall risk". In response to "As a learner, I prefer information from...”, 97\% marked agree or strongly agree when asked about continuing medical education programs, $88 \%$ for professional journals, $63 \%$ for internet resources, and 69\% for consultations with specialists.

Current Implementation of Strategies. Despite the favorable attitude/beliefs and willingness to implement regarding these four fall prevention strategies, there was a significance gap in actual implementation rates (Table 2). Only 19\% of respondents indicated they had received training/education regarding fall prevention in older adults. When asked if there was a balance/strength training exercise program for older adults in their area, $40 \%$ marked yes, $13 \%$ marked no, and $41 \%$ marked unsure.

Table 2. Kansas PAs' attitudes/beliefs and current practices regarding four key fall prevention strategies $(n=152)$.

\begin{tabular}{|c|c|c|c|c|}
\hline & Exercise & Home Safety & Medications & Vision \\
\hline Believe the strategy reduces falls ${ }^{a}$ & $99 \%$ & $99 \%$ & $97 \%$ & $95 \%$ \\
\hline $\begin{array}{l}\text { Believe PAs have a professional } \\
\text { responsibility to implement the strategy }{ }^{\mathrm{a}}\end{array}$ & $95 \%$ & $91 \%$ & $96 \%$ & $88 \%$ \\
\hline $\begin{array}{l}\text { Believe most patients would be compliant } \\
\text { with suggestions regarding the strategy }{ }^{\mathrm{a}}\end{array}$ & $47 \%$ & $69 \%$ & $90 \%$ & $84 \%$ \\
\hline Willing to implement the strategy ${ }^{\mathrm{a}}$ & $93 \%$ & $90 \%$ & $90 \%$ & $85 \%$ \\
\hline Currently implement the strategy ${ }^{\mathrm{b}}$ & $48 \%{ }^{\mathrm{C}}$ & $23 \%$ & $45 \%{ }^{\mathrm{d}}$ & $24 \%$ \\
\hline
\end{tabular}

"Percentage includes those responding as "agree" or "strongly agree”.

"Percentage includes those responding as "frequently" or "always".

"Average of values obtained in response to "ask about exercise habits" (52\%) and "recommend strength/balance programs" (44\%).

dAverage of values obtained in response to "review medications with a focus on fall prevention" (44\%) and "decrease or eliminate medications that potentially increase fall risk" (45\%).

Perceived Barriers. When asked why fall prevention strategies were not implemented routinely in patient encounters, "lack of time” (27\%), "lack of support staff” (26\%), and feeling "ill-prepared to conduct fall screening” (18\%) were marked as barriers.
No respondents marked "not important" and only 1\% marked "not my responsibility" or feeling "ill-prepared to conduct fall focused medication reviews" as barriers. Other potential barriers were identified within the questions asking about difficulties providing 
fall-related preventive care to various populations. Respondents either agreed or strongly agreed that it was difficult to provide fall-related preventive care to patients rarely visiting the practice (86\%), who seem healthy (63\%), have a low socioeconomic status (47\%), or have a different cultural background (41\%).

Logistic Regression. Multiple logistic regression analyses examined factors associated with implementation of the four main fall prevention strategies. The predictor variables chosen for analysis varied depending upon the strategy evaluated (Table 3). Only time was identified as a barrier and independently was associated with implementation of the medication review strategy. When time was not identified as a barrier, respondents were 3.7 times more likely to review medications with a focus on falls. Both time and a balance and strength exercise program in the area were associated positively with implementation of the exercise strategy. When time was not identified as a barrier, respondents were three times more likely to implement the exercise strategy. In addition, when respondents reported there was a balance and strength exercise program in the area, they were four times more likely to implement the exercise strategy.

Table 3. Analysis of predictor variables associated with Kansas PA current practices regarding implementation of four key fall prevention strategies.

\begin{tabular}{|c|c|c|c|c|}
\hline Predictor Variable & $\begin{array}{l}\text { Exercise } \\
\mathbf{n}=137\end{array}$ & $\begin{array}{l}\text { Home Safety } \\
n=139\end{array}$ & $\begin{array}{l}\text { Medications } \\
\mathrm{n}=141\end{array}$ & $\begin{array}{l}\text { Vision } \\
\mathrm{n}=140\end{array}$ \\
\hline $\begin{array}{l}\text { Years in Practice (Reference } \\
\text { Group }=0 \text { - } 4 \text { years) }\end{array}$ & $\mathrm{p}=.788$ & $\mathrm{p}=.526$ & $\mathrm{p}=.379$ & $p=.733$ \\
\hline 5 - 8 years & $\begin{array}{l}1.195 \\
(.42-3.42) \\
p=.740\end{array}$ & $\begin{array}{l}2.103 \\
(.64-6.89) \\
p=.219\end{array}$ & $\begin{array}{l}1.687 \\
(.60-4.75) \\
p=.322\end{array}$ & $\begin{array}{l}1.295 \\
(.38-4.37) \\
p=.677\end{array}$ \\
\hline 9 - 16 years & $\begin{array}{l}1.676 \\
(.62-4.54) \\
p=.309\end{array}$ & $\begin{array}{l}2.308 \\
(.70-7.67) \\
p=.172\end{array}$ & $\begin{array}{l}1.711 \\
(.65-4.54) \\
p=.281\end{array}$ & $\begin{array}{l}1.060 \\
(.32-3.54) \\
p=.924\end{array}$ \\
\hline More than 17 years & $\begin{array}{l}1.255 \\
(.44-3.59) \\
p=.671\end{array}$ & $\begin{array}{l}1.600 \\
(.48-5.36) \\
p=.446\end{array}$ & $\begin{array}{l}2.477 \\
(.89-6.91) \\
p=.083\end{array}$ & $\begin{array}{l}1.810 \\
(.57-5.71) \\
p=.311\end{array}$ \\
\hline Patient will be compliant & $\begin{array}{l}1.246 \\
(.59-2.62) \\
p=.561\end{array}$ & $\begin{array}{l}2.615 \\
(.94-7.28) \\
\mathrm{p}=.066\end{array}$ & $\begin{array}{l}1.354 \\
(.41-4.47) \\
p=.619\end{array}$ & $\begin{array}{l}2.490 \\
(.64-9.70) \\
p=.188\end{array}$ \\
\hline Prior fall prevention training & $\begin{array}{l}1.042 \\
(.39-2.80) \\
p=.934\end{array}$ & $\begin{array}{l}1.990 \\
(.74-5.36) \\
p=.173\end{array}$ & $\begin{array}{l}1.460 \\
(.60-3.58) \\
p=.409\end{array}$ & $\begin{array}{l}2.424 \\
(.91-6.43) \\
p=.075\end{array}$ \\
\hline $\begin{array}{l}\text { Balance and strength program } \\
\text { in the area }\end{array}$ & $\begin{array}{l}4.064 \\
(1.91-8.63) \\
p<.001^{\mathrm{a}}\end{array}$ & NA & NA & NA \\
\hline Barrier: Not enough time & $\begin{array}{l}3.095 \\
(1.25-7.65) \\
p=.014^{\mathrm{a}}\end{array}$ & $\begin{array}{l}3.120 \\
(.96-10.09) \\
p=.057\end{array}$ & $\begin{array}{l}3.7 \\
(1.50-9.11) \\
p=.004^{\mathrm{a}}\end{array}$ & $\begin{array}{l}2.580 \\
(.81-8.20) \\
p=.109\end{array}$ \\
\hline $\begin{array}{l}\text { Barrier: Not prepared to } \\
\text { conduct fall screenings }\end{array}$ & $\begin{array}{l}1.148 \\
(.43-3.08) \\
p=.784\end{array}$ & NA & NA & NA \\
\hline
\end{tabular}

Note: Odds ratio (95\% confidence interval) provided; NA = not applicable due to that predictor not being included in the logistic regression model.

${ }^{a}$ Indicates that the predictor variable was significant. 


\section{Discussion}

Although the majority of respondents expressed positive attitudes expected to be linked with a high level of implementation, most reported they never or only sometimes provide fall-related preventive care to their older adult patients in compliance with the four targeted strategies. This is similar to what has been observed in the literature. ${ }^{7,8}$ As expected, a large number of respondents reported working in rural communities and largely with older adults, indicating the survey likely captured the target respondent population. The top three practice settings reported in this survey were family medicine, orthopedics, and emergency medicine; three common settings where older adults may present following a traumatic fall. Fall prevention programs have been advocated in outpatient clinic settings and also in ED settings. ${ }^{11}$

Many fall prevention strategies rely on improving patients' understanding of fall prevention and lifestyle modification. Therefore, PAs routinely treating older adults should become knowledgeable of not only what they can do to reduce fall risk in their patients, but also identify which members of the healthcare team (e.g., pharmacist, physical/occupation therapists, optometrists) and specific community resources are available to support that goal. The lowest implementation rates reported were for home safety evaluation/referral (23\%) and vision (24\%). During patient encounters, evidence-based practices need to be translated from research ideas to bedside practices. To incorporate these evidencebased practices in patient encounters, strategies to address implementation barriers must be developed at the practitioner level. ${ }^{12}$

A belief that patients will not be compliant might explain a low implementation rate; however, this does not seem to be the case with regards to medication, home safety, and vision, where most respondents believed patients would be compliant, 90\%, 69\%, and 84\%, respectively. Notably, fewer respondents believed patients would be complaint with exercise recommendations (47\%). Beliefs that a particular intervention is ineffective, unimportant, or not one's responsibility were also common barriers that did not appear to be problematic among the PAs in this survey. The majority of respondents reported they believed it was their professional responsibility to implement fall prevention guidelines.

Several barriers to implementation were identified and should be targeted including feeling ill-prepared to conduct fall screening, lack of time, and support staff. Feeling ill-prepared is an internal barrier that could be overcome through broader offering of targeted, fall-related educational programs and continuing medical education (CME). CME should be available to ensure PAs are trained appropriately to provide this type of preventive care. Issues with practice logistics and resources (lack of time and staffing) are more difficult to resolve in a global manner.

One-third of the respondents reported working in rural settings with populations less than 10,000 (24\% of respondents) or 10,000 to 49,000 and not near a metro area (9\%). Fall prevention programs tailored to metro areas or large practice settings within hospitals or full-service clinics likely will not be feasible in rural communities or small clinics with limited resources. Therefore, practical fall prevention programs with materials and tools that are evidence-based, inexpensive or free, quick and easy to implement with the patient and caregivers in communities with limited resources, would be ideal. ${ }^{13}$ For example, such tools might include home safety checklists that can be completed by the patient or caregiver rather than a physical or occupation therapist who may not be available in that county. 
Instructional materials for in-home individual balance/strength programs should be available to distribute to the patient if transportation is an issue or a local program is unaffordable or unavailable. Comprehensive patient/caregiver materials that are interesting, informative, and easy to understand could reduce the one-on-one educational time the PA would have to spend with the patient. Fall risk assessment toolkits are available online (e.g., The Falling LinKS Toolkit). ${ }^{14}$ Lists of community-specific resources (e.g., local fall prevention programs, balance/strength classes, and community organizations that provide home safety evaluation and modification for free or low cost) should be developed and made available.

Development and implementation approaches for these tools and materials as well as provider fall prevention education also should take into account other expressed barriers including how to overcome difficulties in providing preventive care to individuals who rarely visit the practice (e.g., interventions that require less follow-up by the $\mathrm{PA}$ ), of a low socioeconomic status (e.g., low cost or free), and with different cultural backgrounds (e.g., multi-language materials with diverse photos). Interestingly, many PAs reported it is difficult to provide preventive care to patients who seem healthy. As the goal of prevention is to maintain health and independence, this should be re-emphasized with patients to increase awareness and openness to the preventive measures and with the PAs who need to feel comfortable initiating preventive care.

Limitations. The response rate was low. Therefore, non-respondent bias in the results may misrepresent a certain segment of the population who could not or chose not to respond to the survey. However, compared to state data regarding PA practice settings from the American Academy of Physician
Assistants (AAPA) Annual Survey, ${ }^{14}$ our survey yielded a slightly higher than expected percentage of PAs working in family medicine and internal medicine, indicating a sample of the desired population was achieved. AAPA data demonstrated that $43.5 \%$ of Kansas PAs practiced in primary care (defined as family medicine, internal medicine, general pediatrics, and OB/GYN) and $35 \%$ specifically in family medicine. In our sample, $48 \%$ practiced in primary care (family medicine and internal medicine) and $40 \%$ specifically in family medicine. ${ }^{14}$ There also may have been a social desirability bias or reluctance on the part of the participants to give a negative response to current practices and their attitudes/beliefs.

\section{Conclusions}

PAs in Kansas are aware of the importance of fall prevention, believe falls are preventable, and believe it is their professional responsibility to implement fall prevention strategies with their older adult patients. However, most do not implement strategies in their practice due to a variety of logistic and resource factors (lack of time, lack of staff) and internal barriers (feeling ill-prepared). Strategies to bridge the gap between willingness to implement and actual implementation are required. Increased educational opportunities may help PAs feel better prepared to provide targeted fall prevention care to older adults. Access to fall prevention materials, tools, and resources that are practical, simple, inexpensive, and require little implementation time, may overcome some of these barriers and promote the transference of research findings into clinical care.

\section{References}

1 Centers for Disease Control and Prevention and the Merck Company Foundation. The state of aging and 
health in America, 2007. Available at: http://www.cdc.gov/aging/pdf/saha_200 7.pdf.

2 Centers for Disease Control and Prevention, National Center for Injury Prevention and Control, Division of Unintentional Injury Prevention. Falls among older adults: An overview. 2015. Available at: http://www.cdc.gov/HomeandRecreation alSafety/Falls/adultfalls.html. Updated September 20, 2012.

3 Centers for Disease Control and Prevention, National Center for Injury Prevention and Control. Costs of falls among older adults. 2015. Available at: http://www.cdc.gov/injury/wisqars/index .html.

4 National Council on Aging. Falls Free: Promoting a national falls prevention action plan. 2005. Available at: https://www.ncoa.org/wpcontent/uploads/FallsFree_NationalActio nPlan_Final.pdf.

5 American Geriatrics Society and British Geriatrics Society. Summary of the updated American geriatric society/British geriatrics society clinical practice guideline for prevention of falls in older persons. 2010. Available at: http://www.americangeriatrics.org/files/ documents/health_care_pros/JAGS.Falls .Guidelines.pdf.

6 Gillespie LD, Robertson MC, Gillespie $\mathrm{WJ}$, et al. Interventions for preventing falls in older people living in the community. Cochrane Database Syst Rev 2012; 9:CD007146. PMID: 19370674

7 Tinetti ME, Baker DI, King $\mathrm{M}$, et al. Effect of dissemination of evidence in reducing injuries from falls. $\mathrm{N}$ Engl $\mathrm{J}$ Med 2008; 359(3):252-261. PMID: 18635430.

8 Chou WC, Tinetti ME, King MB, Irwin KI, Fortinsky RH. Perceptions of physicians on the barriers and facilitators to integrating fall risk evaluation and management into practice. J Gen Intern Med 2006; 21(2):117-122. PMID: 16336618.

9 Fields A. Discovering Statistics Using SPSS. $3^{\text {rd }}$ edition. Thousand Oaks: Sage Publications; 2009.

10 SPSS. Version 20. Armonk, NY: IBM SPSS Statistics for Windows; 2011.

11 Weigand JV, Gerson LW. Preventive care in the emergency department: Should emergency departments institute a falls prevention program for elder patients? A systematic review. Acad Emerg Med 2001; 8(8):823-826. PMID: 11483459.

12 Titler MG. The evidence for evidence based practice implementation. In: Hughes RG. Patient safety and quality: An evidence-based handbook for nurses. Rockville: Agency for Healthcare Research and Quality (US); 2008. pp. 141.

13 Radebaugh TS, Bahner CA, BallardReisch D, et al. Falling less in Kansas: Development of a fall risk reduction toolkit. J Aging Res 2011: 532079. PMID: 21941655.

14 American Academy of Physician Assistants. State Practice Profiles. 2014. Available at: http://www.aapa.org/WorkArea/Downlo adAsset.aspx?id=1615.

Keywords: physician assistants, attitude, accidental falls, Kansas 